\title{
A study of the factors associated with cervical spinal disc degeneration, with a focus on bone metabolism and amino acids, in the Japanese population: a cross sectional study
}

Kanichiro Wada ${ }^{1,2^{*}}$ (D), Toshihiro Tanaka ${ }^{1}$, Gentaro Kumagai ${ }^{1}$, Hitoshi Kudo ${ }^{1}$, Toru Asari ${ }^{1}$, Daisuke Chiba ${ }^{1}$, Seiya Ota ${ }^{1}$, Keita Kamei ${ }^{1}$, On Takeda', Shigeyuki Nakaji ${ }^{2}$ and Yasuyuki Ishibashi ${ }^{1}$

\begin{abstract}
Background: The physical and biochemical factors responsible for cervical disc degeneration, and resulting in various spinal disorders, remain unclear. This study aimed to evaluate the correlation between cervical spinal canal stenosis and degeneration of intervertebral discs, and to analyze the factors related to disc degeneration in the Japanese population.

Methods: Three hundred and forty-four Japanese general residents underwent investigations, including magnetic resonance imaging of the cervical spine, in our health check project. We measured anteroposterior diameters at the levels of the cervical spinal disc in mid sagittal plane magnetic resonance imaging and evaluated disc degeneration. Spearman correlation coefficient was used to evaluate whether the diameters were correlated with disc degenerative scores. Stepwise multiple linear regression analysis was conducted with the score of disc degeneration as the dependent variable; and age, physical measurement values, bone mineral density of the forearm, and the value of serum bone metabolic markers and amino acids as the independent variables for each sex.

Results: As the age increased, the anteroposterior diameters decreased in both sexes. The minimum anteroposterior diameters were correlated with the disc degenerative scores (Spearman $r=-0.59, p<0.001$ in men, Spearman $r=-0$. $53, p<0.001$ in women). In multiple linear regression analysis, age, cross-linked $\mathrm{N}$-telopeptide of type 1 collagen and isoleucine were significantly correlated with the cervical disc degenerative score in men $\left(R^{2}=0.47\right)$, and age and lysine were significantly correlated with the degenerative score in women $\left(R^{2}=0.50\right)$.
\end{abstract}

Conclusion: The factors responsible for cervical disc degeneration differed between men and women. Whether modifying these significant factors is possible, or whether this intervention would contribute to prevention of disc degeneration requires future studies.

Keywords: Cervical spine, Disc degeneration, Bone metabolism, Amino acid

\footnotetext{
* Correspondence: wadak39@hirosaki-u.ac.jp

${ }^{1}$ Department of Orthopedic Surgery, Hirosaki University Graduate School of

Medicine, Zaifu-cho 5, Hirosaki, Aomori 036-8562, Japan

${ }^{2}$ Department of Social Health, Hirosaki University Graduate School of

Medicine, Zaifu-cho 5, Hirosaki, Aomori 036-8562, Japan
}

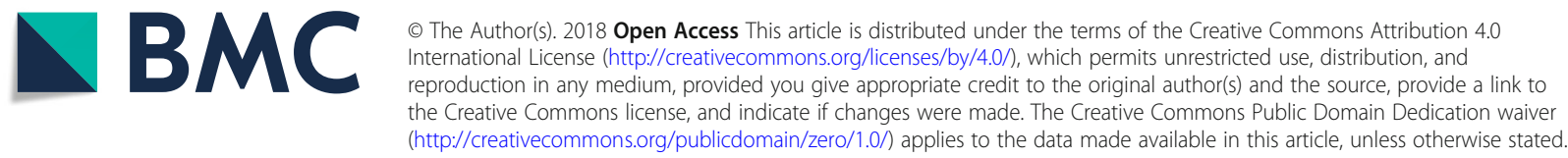




\section{Background}

Cervical myelopathy is a common degenerative spinal disease affecting the activities of daily living [1-3]. One of the pathophysiologies of cervical myelopathy is cervical spinal cord compression, which might be secondary to spinal canal stenosis. Causes of cervical spinal canal stenosis include developmental canal stenosis, intervertebral disc protrusion into the spinal canal, and thickening of the ligamentum flavum [4]. Some cervical degenerative changes are more pronounced in the intervertebral discs, and these changes progress with aging $[5,6]$. In some epidemiologic studies, the prevalence of cervical canal stenosis increased with aging $[7,8]$.

Risk factors of intervertebral disc degeneration are reported to be age $[6,9]$, genetic factors [10], smoking [11], labors [12, 13], and lumbar spine disorders [14, 15]. Although paraspinal muscle atrophy was reported to be associated with lumbar disc degeneration $[16,17]$, no association between cervical muscle and disc degeneration was reported [18]. The relationship between trunk/extremity muscles and cervical disc degeneration remains unclear.

With regards to the biochemical markers of musculoskeletal disorders, some bone metabolism markers were associated with lumbar disc narrowing [19]. One of the useful biomarkers related to cartilage turnover, that might predict progressive knee osteoarthritis, is serum hyaluronic acid which leaks into the blood from degenerated cartilage [20]. Amino acids are chemical constituents of the intervertebral disc [21]; however, there are only a few reports about useful biomarkers for prediction of cervical intervertebral degeneration.

The following issues remain unclear: (1) how are cervical spinal canal stenosis and intervertebral disc degeneration related in each level or overall, and (2) what are the physical features and biochemical makers related to cervical intervertebral degeneration.

The present cross-sectional study aimed to evaluate the correlation between cervical spinal canal stenosis and degeneration of the intervertebral disc, and to analyze whether physical and biochemical data (such as bone metabolism markers and amino acids) relate to cervical intervertebral disc degeneration in the general population.

\section{Methods}

\section{Participants and outline of Iwaki Health Promotion Project}

The Iwaki Health Promotion Project is a health check program designed to improve the average life span. This program was initiated in 2005, and has been conducted annually over a 10-year period. Approximately 1000 adults, aged 20 years and older, living in the Iwaki area of Hirosaki City, Japan, participate every year. In addition to orthopedic specialists, physicians, general surgeons, gynecologists, urologists, psychiatrists, dermatologists, and dentists are involved in this project. As one aspect of the multiple-focused check, we collect biochemical and biomechanical data related to spine disorders, including several parameters of bone metabolism.

A total of 1112 individuals (20-91 years old, 431 men and 681 women) participated in the Iwaki Health Promotion Project in 2015. All participants answered questionnaires about their past medical history, life style, fitness habits, occupational history, family history, and health-related quality of life. They also provided diseasespecific information including a description of their cervical and extremity symptoms. They underwent physical measurements such as height, weight, individual body resistance, etc., and underwent physical examination for assessment of neurological status. In addition, bone mineral density and biochemical examination were performed to evaluate bone status. Participants with a history of stroke, cerebral bleeding, trauma or previous surgery of the cervical spine, systemic diseases involving the cervical spine (e.g., rheumatoid arthritis), and those who did not complete the questionnaires were excluded. Magnetic resonance imaging of the cervical spine was evaluated randomly by age. Finally, 344 people (2086 years old, 151 men and 193 women) were included in our study (Table 1$)$. The mean $( \pm \mathrm{SD})$ ages of the male and female participants were $54.2( \pm 15.1)$ and $55.5( \pm 14$. 6) years, respectively.

\section{Assessment of physical characteristics}

Individual body resistance at $50 \mathrm{kHz}(\mathrm{R})$ was measured using the Tanita MC-190 body composition analyzer (Tanita Co., Tokyo, Japan). Skeletal muscle mass was calculated using Janssen's regression equation, which is based on the relationship between bioelectrical impedance analysis and skeletal muscle mass measured by magnetic resonance imaging [22]. Skeletal muscle mass $(\mathrm{kg})=\left[\left(\right.\right.$ height $\left.^{2} / \mathrm{R} \times 0.401\right)+($ sex $\times 3-825)+($ age $\times-0$. 071) ] + 5.102, where height is the height in centimeters; $\mathrm{R}$ is the resistance in ohms; for sex, men $=1$ and women $=0$; and age is in years. The coefficient of determination $\left(R^{2}\right)$ in this regression equation was 0.86 ; standard error values were $2.7 \mathrm{~kg}$ or $9 \%$. Skeletal muscle index was calculated using skeletal muscle mass $/$ height $^{2} \times 102\left(\mathrm{~kg} / \mathrm{m}^{2}\right)$ for standardizing the differences influenced by height.

\section{Assessment of trunk muscle power}

Trunk-muscle strength was measured using a device consisting of an iron frame combined with a QTM-06b [23]. Isometric trunk-muscle strengths in both extension and flexion were measured as the peak torque $(\mathrm{Nm})$ with the maximum performance of pushing the pad of 
Table 1 Demographic data

\begin{tabular}{|c|c|c|c|}
\hline & Men & Women & $P$ value \\
\hline $\mathrm{N}$ total & 151 & 193 & \\
\hline 20-39 years & 30 & 38 & \\
\hline 40-49 years & 28 & 31 & \\
\hline $50-59$ years & 32 & 37 & \\
\hline $60-69$ years & 33 & 47 & \\
\hline 70- years & 28 & 40 & \\
\hline Age (years) & $54.3 \pm 15.1$ & $55.5 \pm 14.6$ & 0.502 \\
\hline BMI $\left(\mathrm{kg} / \mathrm{m}^{2}\right)$ & $23.9 \pm 3.3$ & $22.2 \pm 3.3$ & 0.000 \\
\hline $\mathrm{SMI}\left(\mathrm{kg} / \mathrm{m}^{2}\right)$ & $18.9 \pm 1.6$ & $15.4 \pm 1.5$ & 0.000 \\
\hline T score of BMD (\%) & $100.0 \pm 10.0$ & $90.2 \pm 16.0$ & 0.000 \\
\hline BAP $(\mu \mathrm{g} / \mathrm{dL})$ & $14.6 \pm 4.6$ & $14.9 \pm 6.2$ & 0.723 \\
\hline NTx (nMBCE/L) & $18.5 \pm 5.3$ & $18.8 \pm 5.9$ & 0.833 \\
\hline TRACP-5b (mU/dL) & $389.9 \pm 124.1$ & $419.9 \pm 197.6$ & 0.312 \\
\hline total P1NP $(\mu \mathrm{g} / \mathrm{L})$ & $48.1 \pm 16.3$ & $55.2 \pm 22.8$ & 0.006 \\
\hline 25(OH)D3 (ng/mL) & $10.7 \pm 7.0$ & $16.2 \pm 6.0$ & 0.000 \\
\hline Pentosidine $(\mathrm{nmol} / \mathrm{mL})$ & $28.0 \pm 9.7$ & $29.8 \pm 12.9$ & 0.224 \\
\hline Isoleuicine (nmol/mL) & $70.4 \pm 14.1$ & $53.5 \pm 8.7$ & 0.000 \\
\hline Threonine $(\mathrm{nmol} / \mathrm{mL})$ & $140.1 \pm 35.5$ & $129.8 \pm 29.3$ & 0.006 \\
\hline Tryptophan (nmol/mL) & $52.7 \pm 7.7$ & $46.6 \pm 6.3$ & 0.000 \\
\hline Valine $(\mathrm{nmol} / \mathrm{mL})$ & $238.3 \pm 36.8$ & $200.9 \pm 30.8$ & 0.000 \\
\hline Histidine (nmol/mL) & $86.1 \pm 9.6$ & $81.1 \pm 7.5$ & 0.000 \\
\hline Phenylalanine (nmol/mL) & $64.1 \pm 10.8$ & $58.2 \pm 9.1$ & 0.000 \\
\hline Methionine (nmol/mL) & $29.0 \pm 5.4$ & $24.9 \pm 3.7$ & 0.000 \\
\hline Lysine (nmol/mL) & $216.6 \pm 33.5$ & $193.8 \pm 28.7$ & 0.000 \\
\hline Leucine $(\mathrm{nmol} / \mathrm{mL})$ & $132.9 \pm 21.4$ & $104.0 \pm 15.2$ & 0.000 \\
\hline
\end{tabular}

Abbreviations: $B M I$ body mass index, SMI skeletal muscle index, $B M D$ bone mineral density, BAP bone-specific alkaline phosphatase, NTX cross-linked $\mathrm{N}$ telopeptide of type 1 collagen, TRACP-5b bone tartrate-resistant acid phosphatase-5b, total P1NP total type 1 procollagen $\mathrm{N}$-terminal propeptide

the measuring device. This torque-value was adjusted for the body weight $(\mathrm{Nm} / \mathrm{kg})$.

\section{Assessment of magnetic resonance imaging}

A 1.5-Tesla superconducting imager and phased array coils was used for all subjects in the present study. A mobile magnetic resonance imaging unit (Intera Achieva 1.5-Tesla; Philips, Amsterdam, Nederland) was used. The cervical spine magnetic resonance imaging was performed with participants in the supine position. The imaging protocol included sagittal T2-weighted fast-spin echo (repetition time, $4000 \mathrm{~ms} / \mathrm{echo}$; echo time, $200 \mathrm{~ms}$; field of view, $300 \times 320 \mathrm{~mm}$ ) and axial T2-weighted fastspin echo (repetition time, $4000 \mathrm{~ms} / \mathrm{echo}$; echo time, $120 \mathrm{~ms}$; field of view, $180 \times 180 \mathrm{~mm}$ ). Sagittal T2weighted images were used to assess the intervertebral space from $\mathrm{C} 2 / 3$ to $\mathrm{C} 7 / \mathrm{T} 1$.
Evaluation of intervertebral disc degeneration on cervical magnetic resonance imaging

Intervertebral disc degeneration was evaluated based on the following two magnetic resonance imaging findings: (1) decrease in the signal intensity of the intervertebral discs, and (2) disc space narrowing. The cervical intervertebral levels evaluated for disc degeneration included all levels from $\mathrm{C} 3 / 4$ to $\mathrm{C} 7 / \mathrm{T} 1$. To rate the magnetic resonance findings, we used Matsumoto's classification [5]. The scoring system for the different magnetic resonance findings was as follows; decrease in signal intensity of intervertebral disc; grade 0: as hyperintense as or slightly less bright than cerebrospinal fluid, grade 1: markedly hypointense than cerebrospinal fluid, and grade 2: no signal. Disc space narrowing; grade 0: $100-75 \%$ of height of upper healthy disc, grade 1: $75-50 \%$ of height of upper healthy disc, and grade 2: less than $50 \%$ of height of upper healthy disc.

In addition, to determine the severity of cervical disc degeneration, the examiners summed the values of Matsumoto's classification in each of the intervertebral sections. This summed value was defined as the severity score of cervical disc degeneration. According to this severity score, 0 corresponded to normal cervical disc and 20 to most severe degenerative cervical disc (Fig. 1).

In our preliminary data from 50 adults (mean age: 55.9 \pm 16.6 years old), the intra-and inter-reliabilities of evaluating the decrease in signal intensity of the intervertebral discs/disc space narrowing on cervical magnetic resonance imaging were as follows: intra-observer agreements for WK (spine surgeon with 19-year experience) and OS (orthopedic surgeon with 5-year experience) were excellent (kappa $=0.839-0.913$ ), and inter-observer agreements were moderate $(\mathrm{kappa}=0.555-0.586)$.

\section{Measurement of anterior-posterior diameter of the cerebrospinal fluid column on mid-sagittal magnetic resonance imaging}

We used a mid-sagittal magnetic resonance imaging scan to measure the anterior-posterior diameter of the cerebrospinal fluid column at the $\mathrm{C} 3 / 4, \mathrm{C} 4 / 5, \mathrm{C} 5 / 6, \mathrm{C} 6 /$ 7 , and C7/T1 intervertebral levels. In addition, the value obtained by dividing the anterior-posterior diameter of the cerebrospinal column by the mid-sagittal anteriorposterior diameter of $\mathrm{C} 3$ vertebral body was defined as $\%$ anterior-posterior diameter (Fig. 2).

The inter- and intra-observer agreements of cerebrospinal fluid column measurement from 50 adults were as follows: intra-class correlation coefficient [ICC $(1,1)]$ for WK was 0.734 (95\% confidence interval (CI): 0.575-0.839; $P \leq 0.001$ ), and for OS was 0.818 (95\% CI: $0.680-0.896$; $P \leq 0.001)$. Interclass correlation coefficient $[\operatorname{ICC}(2,1)]$ in WK and OS was 0.623 (95\% CI: $0.413-0.768 ; P \leq 0.001$ ). 


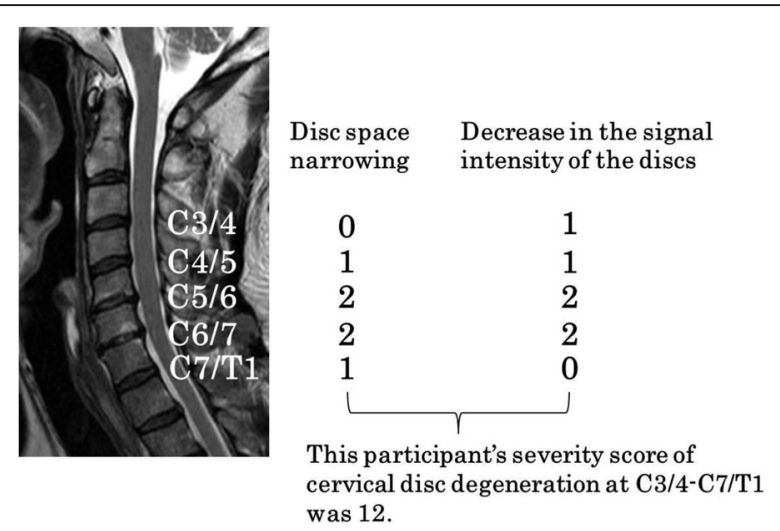

Fig. 1 Evaluation of severity score of cervical spondylosis. Intervertebral disc degeneration was evaluated based on the following two magnetic resonance findings: (1) decrease in the signal intensity of the intervertebral discs, and (2) disc space narrowing. The cervical intervertebral levels evaluated for disc degeneration included all levels from C3/4 to C7/T1. To rate the magnetic resonance findings, we used Matsumoto's classification [Matsumoto M, JBJS-Br 1998]

\section{Assessment of bone status, serum bone metabolic markers and essential amino acids}

Dual energy X-ray absorptiometry of forearm was measured and the bone mineral density scores were calculated as T-score. Blood samples were collected in the early morning, and these were immediately centrifuged. Serum samples were extracted and transferred to a deep freezer. Serum bone-specific alkaline phosphatase $(\mu \mathrm{g} / \mathrm{dL})$ was quantified using a chemiluminescent enzyme immunoassay/antibody radioimmunoassay (LSI Medience Corp., Tokyo, Japan). Serum pentosidine concentration (pmol/ $\mathrm{mL}$, high performance liquid chromatography; LSI Medience Corp., Tokyo, Japan), undercarboxylated osteocalcin (ng/mL,:electro-chemiluminescence immunoassay; LSI Medience Corp., Tokyo, Japan), cross-linked N-telopeptide of type 1 collagen (nMBCE/L, enzyme immunoassay; LSI Medience Corp., Tokyo, Japan), bone tartrate-resistant acid phosphatase-5b (mU/dL, enzyme immunoassay; LSI Medience Corp., Tokyo, Japan), total type 1 procollagen $\mathrm{N}$ terminal propeptide $(\mu \mathrm{g} / \mathrm{dL}$, electro-chemiluminescence immunoassay; LSI Medience Corp., Tokyo, Japan), 25(OH) D3 (ng/mL, liquid chromatography-tandem mass spectrometry; LSI Medience Corp., Tokyo, Japan), and essential amino acids (tryptophan, lysine, methionine, threonine, valine, leucine, isoleucine, and histidine; $\mathrm{nmol} / \mathrm{mL}$, high performance liquid chromatography) were measured.

\section{Statistical analysis}

The data were analyzed using SPSS ver. 22.0 (SPSS Inc., Chicago, IL, USA). All data were grouped according to sex.

For multiple group comparisons of \% anterior-posterior diameters and disc degenerative scores between age groups, we used analysis of variance. Statistical significance

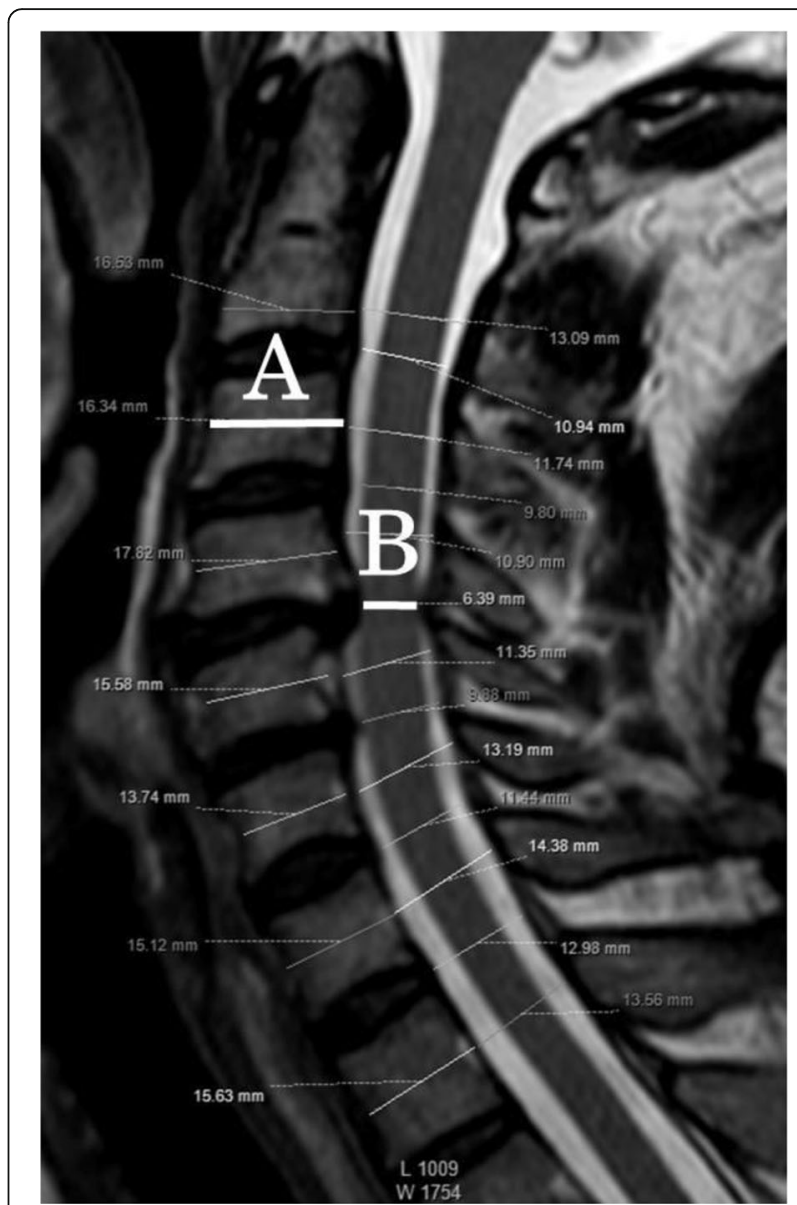

Fig. 2 Measurement of the diameter on mid-sagittal magnetic resonance imaging. Minimum anterior-posterior diameter on mid-sagittal magnetic resonance imaging / anterior-posterior diameter of C3 vertebral body was calculated by dividing B by $A$ as illustrated in this figure. $\mathrm{A}$ is the anterior-posterior diameter of C3 vertebral body, B is the minimum anterior-posterior diameter of spinal canal from $\mathrm{C} 3 / 4$ to $\mathrm{C} 7 / \mathrm{T} 1$

of individual differences was evaluated using the Tukey's honestly significant difference test if analysis of variance was significant. The \% anterior-posterior diameter and the disc degenerative score were analyzed for each disc level.

Spearman correlation coefficient was used to evaluate whether \% anterior-posterior diameter at each level was correlated with the severity score of cervical disc degeneration.

For correlations between the severity score of cervical disc degeneration and other parameters: age, body mass index, skeletal muscle index, exercise habit, $\mathrm{T}$ score of bone mineral density, and the serum values (bone-specific alkaline phosphatase, pentosidine, cross-linked $\mathrm{N}$ telopeptide of type 1 collagen, bone tartrate-resistant acid phosphatase-5b, $25(\mathrm{OH}) \mathrm{D} 3$, isoleucine, threonine, tryptophan, valine, histidine, phenylalanine, methionine, lysine, and leucine); Spearman correlation coefficient 
was used. We conducted stepwise multiple regression analyses with the severity score of cervical disc degeneration as the dependent variable and parameters showing significant correlation by single correlation analysis as independent variables.

$P$ values $<0.05$ were considered statistically significant.

\section{Results}

Comparison of \% anterior-posterior diameters and disc degenerative scores between age groups

In men, $\%$ anterior-posterior diameter was significantly reduced with aging at the levels of $\mathrm{C} 3 / 4, \mathrm{C} 4 / 5, \mathrm{C} 5 / 6$, C6/7 and minimum diameter from the $20 \mathrm{~s}$ and $30 \mathrm{~s}$, to 60 s age groups (Fig. 3). Almost the same trend was observed in women (Fig. 4). The disc degeneration score increased significantly as the participants became older for both sexes. Severity score of the disc degeneration was doubled in the 50-59 years group as compared to that in the 20-39 age group in men (Fig. 5) and women (Fig. 6). In both sexes, \% anterior-posterior diameter was the smallest, and disc degenerative score was the lowest at $\mathrm{C} 5 / 6$.

\section{Correlation of spinal canal diameter with disc degeneration score}

There was a significant negative correlation between \% anterior-posterior diameters and disc degenerative scores at all levels in both sexes. Values of correlation coefficients ranged from -0.646 to -0.325 in the same intervertebral levels. Values of correlation coefficients between the diameter in each intervertebral level and the severity score of cervical disc degeneration ranged from -0.376 to -0.625 . The correlation between the minimum \% anterior-posterior diameter and the severity score of cervical disc degeneration was -0.593 in men and -0.527 in women (Tables 2 and 3).

\section{Correlation of the cervical disc degeneration with} physical, bone status and biochemical markers, and multiple regression models for elucidating the factors correlated with the cervical disc degeneration on magnetic resonance imaging

The severity score of the cervical disc degeneration was significantly correlated with age, trunk-muscle strength in flexion, pentosidine, cross-linked N-telopeptide of type 1 collagen, and leucine in men (Table 2); and with age, body mass index, skeletal muscle index, trunkmuscle strength in flexion, $\mathrm{T}$ score of bone mineral density, pentosidine, bone tartrate-resistant acid phosphatase-5b, cross-linked N-telopeptide of type 1 collagen, undercarboxylated osteocalcin, 25(OH)D3, lysine in women (Table 3).

In multiple linear regression analysis, age, cross-linked $\mathrm{N}$-telopeptide of type 1 collagen and isoleucine were significantly correlated with the total cervical degeneration score in men $\left(R^{2}=0.470\right.$, Table 4$)$, and age and lysine were significantly correlated with the total cervical degeneration score in women $\left(R^{2}=0.500\right.$, Table 5$)$.

\section{Discussion}

The present cross-sectional study evaluated the correlation between cervical canal stenosis and degeneration of the intervertebral disc in the general population, and analyzed factors related to cervical intervertebral disc degeneration. With aging, the cervical canal diameters became smaller and the cervical disc degeneration scores became higher. Kato et al. reported that spinal dural tube diameter tended to decrease with age, especially at the intervertebral disc levels, and showed that

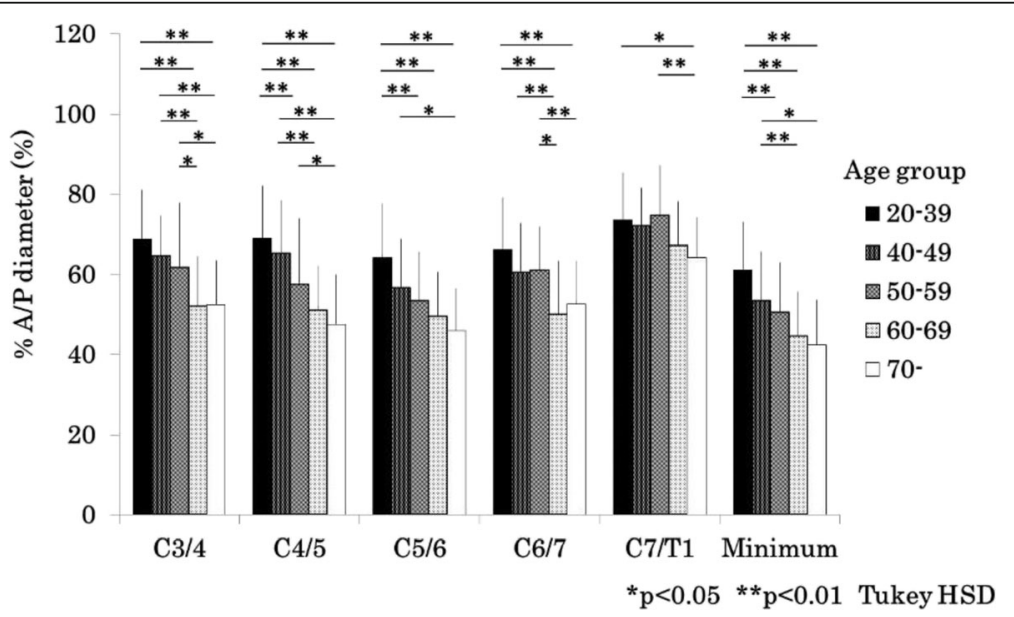

Fig. 3 Bar chart (mean and standard deviation) showing the \% anterior-posterior diameter by age at each level and minimum \% anterior-posterior diameter in men. A/P: anterior-posterior, HSD: honestly significant difference 


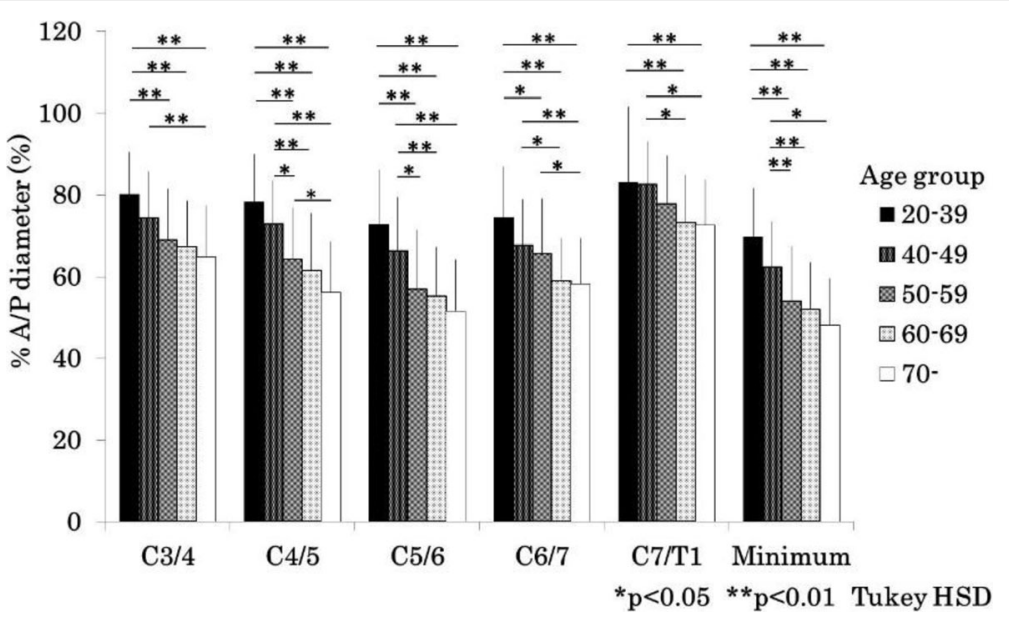

Fig. 4 Bar chart (mean and standard deviation) showing the \% anterior-posterior diameter by age at each level and minimum \% anterior-posterior diameter in women. A/P: anterior-posterior, HSD: honestly significant difference

degenerative changes in the cervical spine progressed chiefly in the intervertebral discs [8]. In a previous report, prevalence of cervical cord compression was higher in men than in women [7]. In the current study, spinal canal anterior-posterior diameter normalized by $\mathrm{C} 3$ vertebral body was larger in women than in men. In addition, the smallest relative diameter was at $\mathrm{C} 5 / 6$, followed by $\mathrm{C} 4 / 5$ and $\mathrm{C6} / 7$. This was in line with previous reports showing that spinal cord compression was most frequent at $\mathrm{C} 5 / 6[7,24]$.
A significant negative correlation was found between \% anterior-posterior diameters and disc degeneration score at all levels in both sexes. Morishita et al. reported that disc degeneration grades were worse in symptomatic subjects with two-level cervical segments stenosis than in those with one-level stenosis [25]. They proposed that multi-level cervical stenosis may develop following initial stenosis at the C5/6 segment. In this study, disc degeneration score at $\mathrm{C} 5 / 6$ was the most advanced in both sexes.

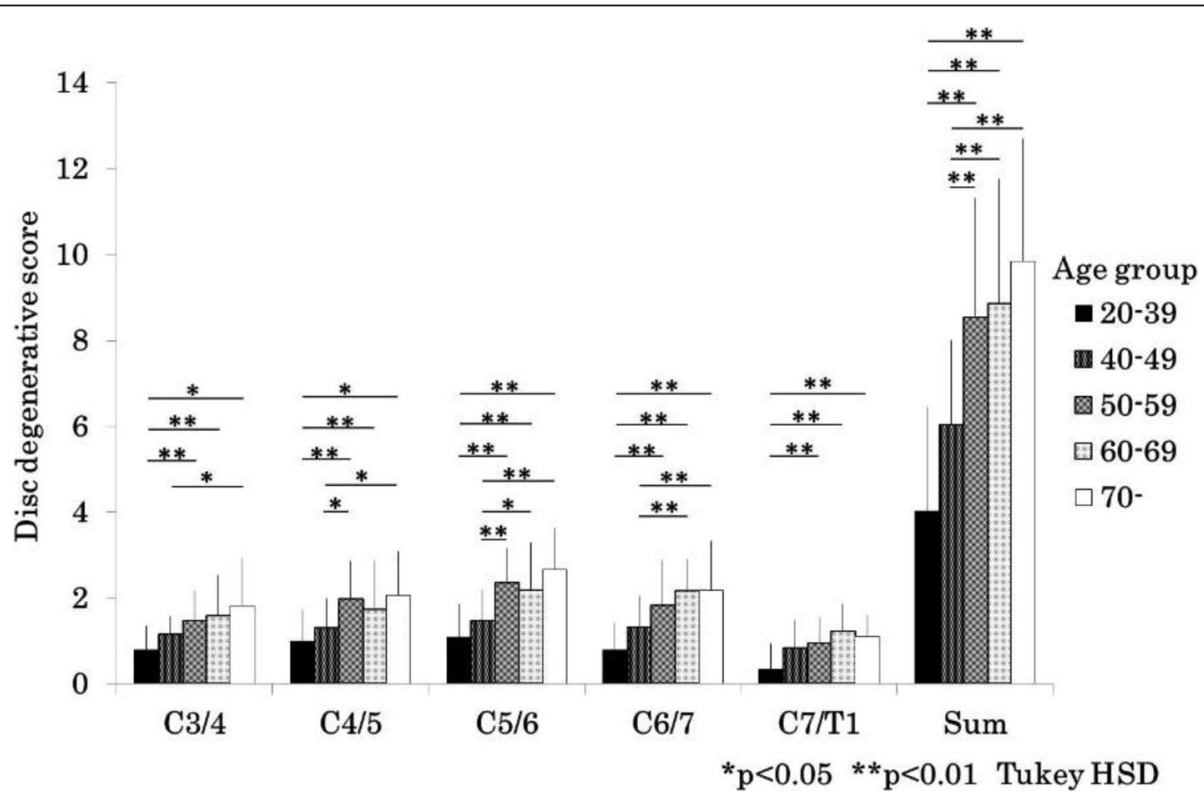

Fig. 5 Bar chart (mean and standard deviation) showing disc degenerative score by age at each level and sum of score in men. HSD: honestly significant difference 


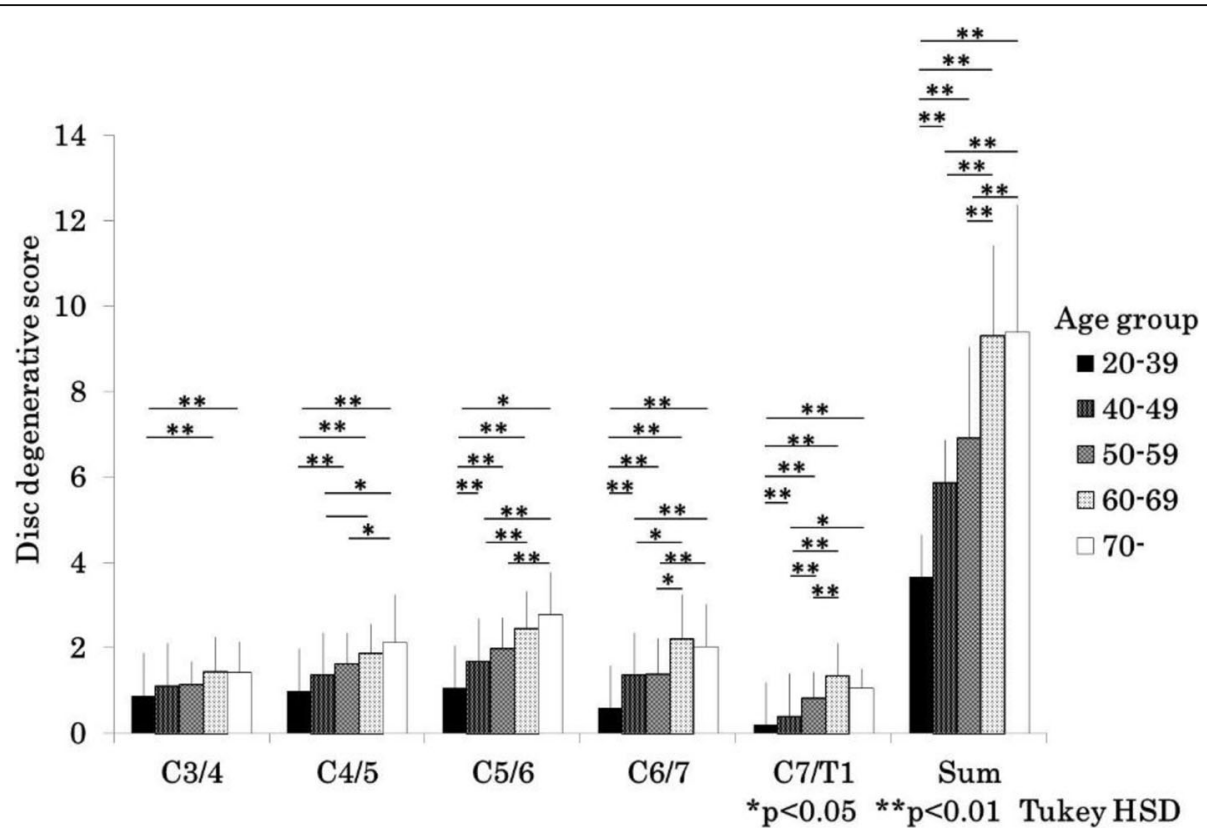

Fig. 6 Bar chart showing (mean and standard deviation) the disc degenerative score by age at each level and sum of score in women. HSD: honestly significant difference

In Spearman correlation coefficient, age was moderately correlated with the severity score of cervical disc degeneration in both sexes, age explained $>40 \%$ of the variance in men and $>45 \%$ in women. The other factors explained from 2 to $28 \%$. Omair reported that age was the most significant determinant of lumbar disc degeneration in their genetic association study [26]. Age might also be the strongest factor related to cervical disc degeneration in our current study. In multiple linear regression analysis, age, cross-linked $\mathrm{N}$-telopeptide of type 1 collagen and isoleucine were significantly correlated with the total cervical degeneration score in men $\left(R^{2}=0.470\right.$, Table 4$)$, while age and lysine were significantly correlated with the total cervical degenerative score in women $\left(R^{2}=0.500\right.$, Table 5). Goode reported a positive correlation, adjusted for sex, between severity of lumber disc space narrowing and urinary cross-linked $\mathrm{N}$ telopeptide of type 1 collagen [19]. Thoracic spinal disc space narrowing was one of the risk factors for vertebral fractures in men over 50 years old [27]. Only a few studies reported the relation between bone turnover and osteoporosis in men, and Goode's data were opposite to this survey, which showed a negative correlation between spinal disc degeneration and serum cross-linked $\mathrm{N}$-telopeptide of type 1 collagen. However, these previous reports did not look at the cervical spine specifically. In the present study, there was a significant negative correlation between age and cross-linked N- telopeptide of type 1 collagen under 60 years of age ( $\rho$ $=-0.297, P=0.004)$, while there was a positive correlation over 60 years of age $(\rho=0.282, P=0.027)$ in men (Fig. 7). This trend was similar to the longitudinal data reported by Yoshimura et al. Furthermore, disc degenerations were more severe at 50-59 years of age than below 50 years, and there was no difference between 50 and 59 years of age and over 60 years. There was a significant correlation between disc degenerative score and age, isoleucine, tryptophan, valine, and leucine below 60 years of age. Over 60 years of age, only $25(\mathrm{OH})$ D3 was significantly correlated with disc degeneration scores (Table 6). Factors related to disc degeneration may differ according to age.

Isoleucine, valine and leucine are branched chain amino acids. Branched chain amino acids are one of the disc components [21]. Leucine intake was associated with long-term lean body mass retention [28]. Disc degeneration was positively correlated with paraspinal muscle atrophy and fatty infiltration [16, 17], and muscle strength had a greater impact on disc degeneration [29] at the lumbar spine. Okada reported that there was no significant correlation between cervical disc degeneration and cross-sectional area of the posterior cervical extensor muscles [14]. In the current study, trunk muscle strength in flexion was associated with cervical disc degeneration in univariate correlation. Trunk muscles strength may be related to cervical disc degeneration. Polymorphisms 


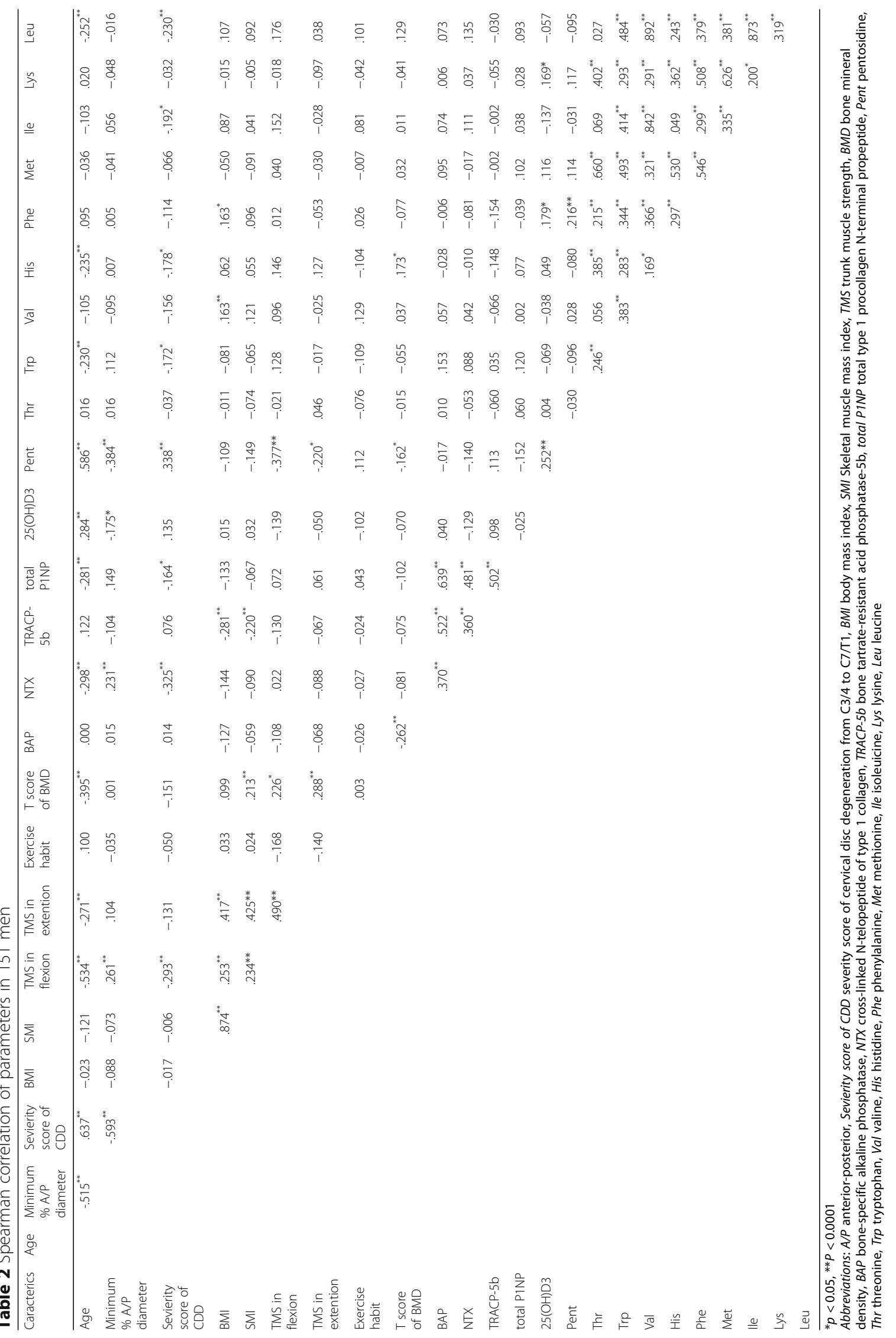




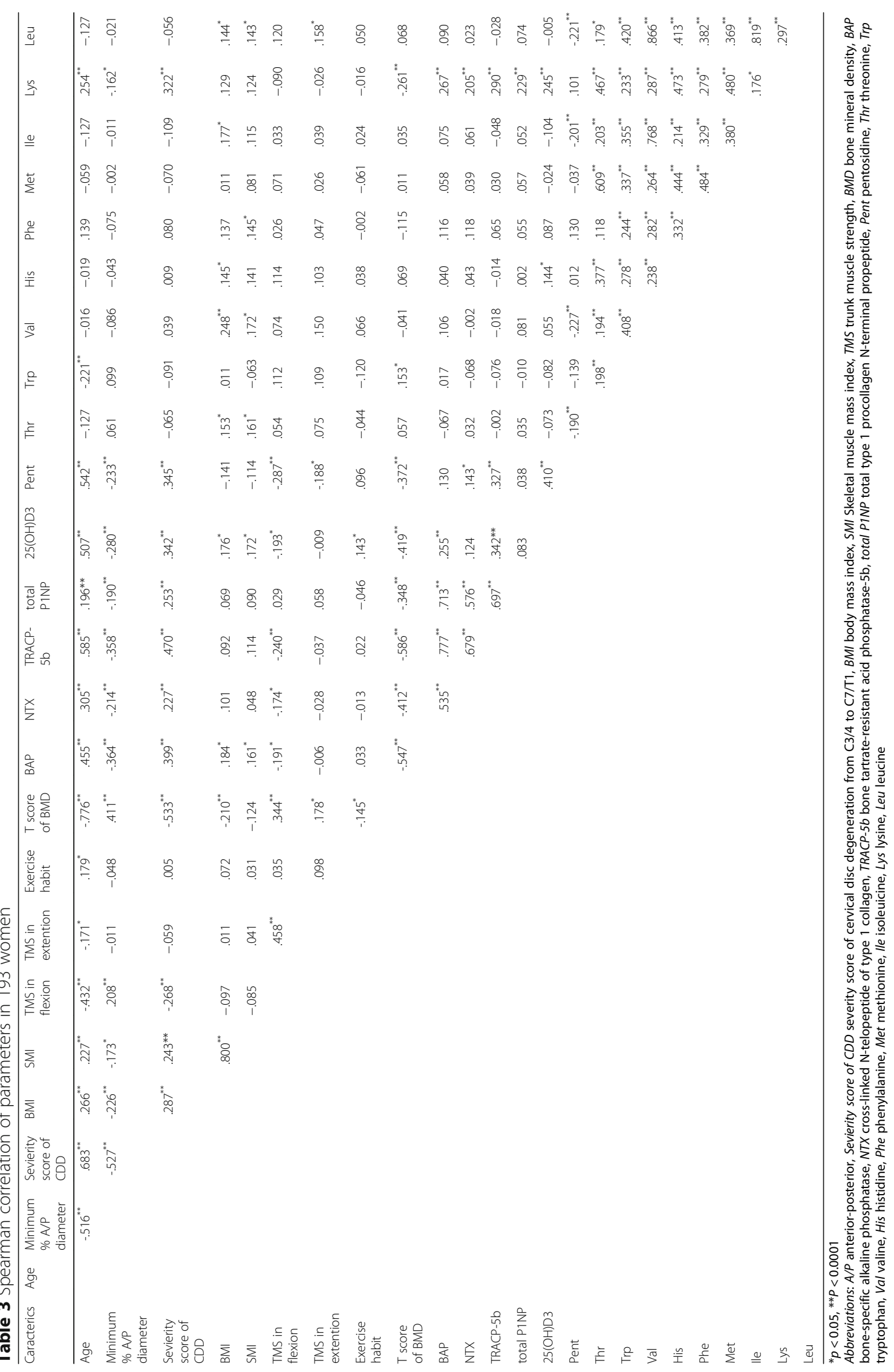


Table 4 Analysis of the factors correlated with the severity score of cervical disc degeneration on magnetic resonance imaging in men

\begin{tabular}{lllll}
\hline $\mathrm{R}^{2}=0.470$ & $\mathrm{~B}$ & $\beta$ & $\mathrm{P}$ value & $95 \% \mathrm{Cl}$ \\
\hline Age & 0.119 & 0.557 & $<0.001$ & 0.089 to 0.149 \\
NTX & -0.094 & -0.156 & 0.027 & -0.177 to -0.011 \\
Isoleucine & -0.045 & -0.181 & 0.008 & -0.078 to -0.012
\end{tabular}

Abbreviations: $\mathrm{Cl}$ confidence interval, NTX cross-linked $\mathrm{N}$-telopeptide of type 1 collagen

were found in the gene encoding vitamin $\mathrm{D}$ receptor [30, 31]. A longitudinal study is required to clarify whether there is a relation between the ingestion of branched chain amino acids and increasing bone turnover in individuals younger than 60 years, and whether ingestion of vitamin D3 in those over 60 years of age can prevent progression of disc degeneration. In women, lysine may be a biomarker for disc degeneration. In human thoracic discs, the content of pyridinoline decreased with increasing age [32], and lysine was associated with collagen crosslinkage [33]. The association between serum lysine and collagen cross-linking metabolites remain unclear. Longitudinal research is necessary to clarify the usefulness of lysine as a biomarker for disc degeneration.

The present study has several limitations. First, it was a cross sectional study, and therefore, the rate of progression of disc degeneration could not be clarified. Second, our survey was limited to Japanese subjects, and hence possible ethnic differences were not considered. Third, in our participants the proportion of smoking habits (men: 60.9\% current and exsmokers, $21.9 \%$ current smokers; women: $20.7 \%$ current and ex-smokers, 9.3\% current smokers) may be lower than that in the general population as is the prevalence of diabetes (men: $7.9 \%$, women: $4.6 \%$ ). The subjects of this survey included those receiving osteoporosis treatment (men: 0.7\%, women: 4.1\%). These conditions may affect disc degeneration and/or bone metabolism. Fourthly, qualitative assessment of disc space narrowing might be difficult in patients with multiple vertebral disc reductions. However, there was

Table 5 Analysis of the factors correlated with the severity score of cervical disc degeneration on magnetic resonance imaging in women

\begin{tabular}{lllll}
\hline $\mathrm{R}^{2}=0.500$ & $\mathrm{~B}$ & $\beta$ & $P$ value & $95 \% \mathrm{Cl}$ \\
\hline Age & 0.147 & 0.658 & $<0.001$ & 0.121 to 0.173 \\
Lysine & 0.015 & 0.136 & 0.023 & 0.002 to 0.027 \\
\hline
\end{tabular}

Abbreviation: $\mathrm{Cl}$ confidence interval

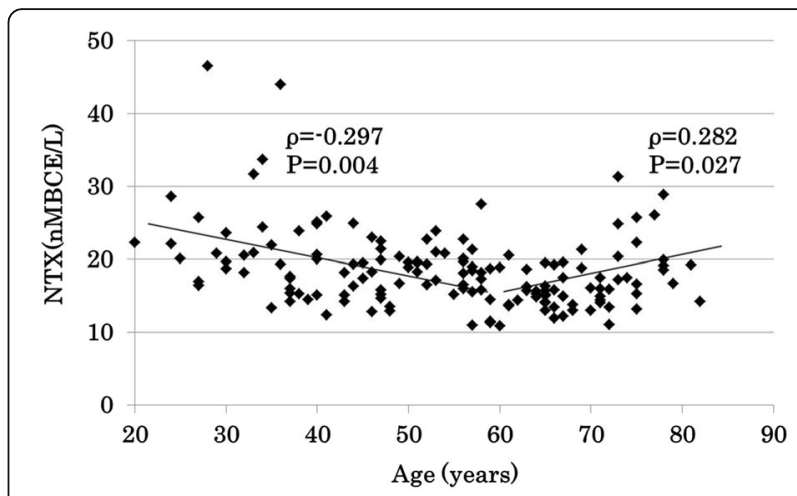

Fig. 7 Scatter plot showing the correlation between age and crosslinked N-telopeptide of type 1 collagen in men. NTX: cross-linked $\mathrm{N}$-telopeptide of type 1 collagen

an acceptable validation for the evaluation of the disc degeneration on MRI in this study; therefore this limitation is not expected to have a major impact on the analysis.

\section{Conclusions}

The anteroposterior diameters of cervical spine on magnetic resonance imaging decreased with age in both sexes. The anteroposterior diameters were correlated to disc degeneration. In multiple linear regression analysis, age, cross-linked $\mathrm{N}$-telopeptide of type 1 collagen and isoleucine were significantly correlated with the cervical disc degenerative score in men, while age and lysine were significantly correlated with the degenerative score in women. Factors contributing to cervical disc degeneration were different between men and women. Whether modifying these significant factors is possible, or whether this intervention would contribute to prevention of disc degeneration requires future studies.

Table 6 Correlation coefficients between disc degenerative score and parameters in men under 60 years old and over 60 years old

\begin{tabular}{llll}
\hline Age & parameters & $\rho$ & P value \\
\hline Under 60 years & Age & 0.668 & $<0.001$ \\
& NTX & -0.380 & $<0.001$ \\
& Isoleucine & -0.352 & 0.001 \\
& Tryptophan & -0.252 & 0.017 \\
& Valine & -0.255 & 0.033 \\
Over 60 years & Leucine & -0.303 & 0.004 \\
\hline
\end{tabular}

Abbreviation: NTX cross-linked N-telopeptide of type 1 collagen 


\section{Acknowledgements}

We would like to thank Editage (www.editage.jp) for English language editing.

\section{Funding}

This work was supported by JOA-Subsidized Science Project Research from the Japanese Orthopedic Association (No. 2015-2), the Center of Innovation Program from Japan Science and Technology Agency, JST (No. 2014-377), and a Japanese Health Labor Sciences Research Grant.

\section{Availability of data and materials}

The datasets used and/or analysed during the current study are available from the corresponding author on reasonable request.

\section{Authors' contributions}

Conceived and designed the experiments: KW GK SN YI. Performed the experiments: KW TT GK HK TA DC SO KK OT. Analyzed the data: KW DC SO. Wrote the paper: KW. Revised the paper critically for important intellectual content: TT GK HK TA. All authors read and approved the final manuscript.

\section{Ethics approval and consent to participate}

For this cross-sectional survey, the ethics committee of Hirosaki University, Graduate School of Medicine approved the study (2014-377), and all participants provided written informed consent before participation.

\section{Competing interests}

The authors declare that they have no competing interests.

\section{Publisher's Note}

Springer Nature remains neutral with regard to jurisdictional claims in published maps and institutional affiliations.

\section{Received: 29 July 2017 Accepted: 23 April 2018}

\section{Published online: 17 May 2018}

\section{References}

1. Nurick $\mathrm{S}$. The pathogenesis of the spinal cord disorder associated with cervical spondylosis. Brain. 1972;95:87-100

2. Nurick $\mathrm{S}$. The natural history and the results of surgical treatment of the spinal cord disorder associated with cervical spondylosis. Brain. 1972;95:1018.

3. Iwasaki M, Kawaguchi Y, Kimura T, Yonenobu K. Long-term results of expansive laminoplasty for ossification of the posterior longitudinal ligament of the cervical spine: more than 10 years follow up. J Neurosurg. 2002;96:180-9.

4. Muhle C, Metzner J, Weinert D, Falliner A, Brinkmann G, Mehdom MH, et al. Classification system based on kinematic MR imaging in cervical spondylitic myelopathy. Am J Neuroradiol. 1998;19:1763-71.

5. Matsumoto M, Fujimura Y, Suzuki N, Nishi Y, Nakamura M, Yabe Y, et al. MRI of cervical intervertebral discs in asymptomatic subjects. J Bone Joint Surg Br. 1998;80:19-24.

6. Okada E, Matsumoto M, Ichihara D, Chiba K, Toyama Y, Momoshima S, et al. Aging of the cervical spine in healthy volunteers: a 10-year longitudinal magnetic resonance imaging study. Spine. 2009;34:706-12.

7. Nagata K, Yoshimura N, Muraki S, Hashizume H, Ishimoto Y, Yamada H, et al. Prevalence of cervical cord compression and its association with physical performance in a population-based cohort in Japan. Spine. 2012;37:1892-8.

8. Kato F, Yukawa Y, Suda K, Yamada M, Ueta T. Normal morphology, agerelated changes and abnormal findings of the cervical spine. Part II: magnetic resonance imaging of over 1,200 asymptomatic subjects. Eur Spine J. 2012;21:1499-507.

9. Kumagai G, Ono A, Numasawa T, Wada K, Inoue R, Iwasaki H, et al. Association between roentgenographic findings of the cervical spine and neck symptoms in a Japanese community population. J Orthop Sci. 2014;19:390-7.

10. Sambrook PN, MacGregor AJ, Spector TD. Genetic influences on cervical and lumbar disc degeneration: a magnetic resonance imaging study in twins. Arthritis Rheum. 1999;42:366-72.

11. Brain WR. Discussion of rupture of the intervertebral disc in the cervical region. Proc R Soc Med. 1948;49:509-16.

12. Hagberg M, Wegman DH. Prevalence rates and odds ratios of shoulder-neck diseases in different occupational groups. Br J Ind Med. 1987;44:602-10.
13. Takamiya Y, Nagata K, Fukuda K, Shibata A, Ishitake T, Suenaga T. Cervical spine disorders in farm workersrequiring neck extension actions. J Orthop Sci. 2006;11:235-40.

14. Okada E, Matsumoto M, Fujiwara $H$, Toyama Y. Disc degeneration of cervical spine on MRI in patients with lumbar disc herniation: comparison study with asymptomatic volunteers. Eur Spine J. 2011;20:585-91.

15. Matsumoto M, Okada E, Toyama Y, Fujiwara H, Momoshima S, Takahata T. Tandem age-related lumbar and cervical intervertebral disc changes in asymptomatic subjects. Eur Spine J. 2013;22:708-13.

16. Sun D, Liu P, Cheng J, Ma Z, Liu J, Qin T. Correlation between intervertebral disc degeneration, paraspinal muscle atrophy, and lumbar facet joints degeneration in patients with lumbar disc herniation. BMC Musculoskelet Disord. 2017;18:167.

17. Teichtahl AJ, Urquhart DM, Wang Y, Wluka AE, O'Sullivan R, Jones G, et al. Lumbar disc degeneration is associated with modic change and high paraspinal fat content - a 3.0T magnetic resonance imaging study. BMC Musculoskelet Disord. 2016;17:439.

18. Okada E, Matsumoto M, Ichihara D, Chiba K, Toyama Y, Fujiwara H, et al. Cross-sectional area of posterior extensor muscles of the cervical spine in asymptomatic subjects: a 10-year longitudinal magnetic resonance imaging study. Eur Spine J. 2011;20:1567-73.

19. Goode AP, Marshall SW, Kraus VB, Renner JB, Stürmer T, Carey TS, et al. Association between serum and urine biomarkers and lumbar spine individual radiographic features: the Johnston County Osteoarthritis Project. Osteoarthr Cartil. 2012;20:1286-93.

20. Sasaki E, Tsuda E, Yamamoto Y, Maeda S, Inoue R, Chiba D, et al. Serum hyaluronic acid concentration predicts the progression of joint space narrowing in normal knees and established knee osteoarthritis - a five-year prospective cohort study. Arthritis Res Ther. 2015;17:283.

21. Radek M, Pacholczyk-Sienicka, Jankowski, Albrecht L, Grodzka M, Depta A, et al. Assessing the correlation between the degree of disc degeneration on the Pfirrmann scale and the metabolites identified in HR-MAS NMR spectroscopy. Magn Reson Imaging. 2016;34:376-80.

22. Janssen I, Heymsfield SB, Baumgartner RN, Ross R. Estimation of skeletal muscle mass by bioelectrical impedance analysis. J Appl Physiol. 2000;89:465-71.

23. Chiba D, Tsuda E, Wada K, Kumagai G, Sasaki E, Nawata A, et al. Lumbar spondylosis, lumbar spinal stenosis, knee pain, back muscle strength are associated with the locomotive syndrome: rural population study in Japan. $J$ Orthop Sci. 2016;21:366-72.

24. Nakashima H, Yukawa Y, Suda K, Yamada M, Ueta T, Kato F. Abnormal findings on magnetic resonance images of the cervical spines in 1211 asymptomatic subjects. Spine. 2015;40:392-8.

25. Morishita $Y$, Naito M, Wang JC. Cervical spinal canal stenosis: the differences between stenosis at the lower cervical and multiple segment levels. Int Orthop. 2011;35:1517-22.

26. Omair A, Mannion AF, Holden M, Leivseth G, Fairbank J, Hägg O, et al. Age and pro-inflammatory gene polymorphisms influence adjacent segment disc degeneration more than fusion does in patients treated for chronic low back pain. Eur Spine J. 2016;25:2-13.

27. Pariente E, Olmos JM, Landeras R, Nan D, González-Macias J, Hemández JL. Relationship between spinal osteoarthritis and vertebral fractures in men older than 50 years: data from the Camargo Chort Study. J Bone Miner Metab. 2017:35:114-21.

28. McDonald CK, Ankarfeldt MZ, Capra S, Bauer J, Raymond K, Heitmann BL. Lean body mass change over 6 years is associated with dietary leucine intake in an older Danish population. Br J Nutr. 2016;115:1556-62.

29. Battie MC, Videman T, Kaprio J, Gibbons LE, Gill K, Manninen H, et al. The twin spine study: contributions to a changing view of disc degeneration. Spine J. 2009;9:47-59.

30. Kawaguchi Y, Kanamori M, Ishihara H, Ohmori K, Matsui H, Kimura T. The association of lumbar disc disease with vitamin-D receptor gene polymorphism. J Bone Joint Surg Am. 2002;84:2022-8.

31. Videman T, Leppavuori J, Kaprio J, Battié MC, Gibbons LE, Peltonen L, et al. Intragenic polymorphisms of the vitamin $D$ receptor gene associated with intervertebral disc degeneration. Spine. 1998;23:2477-85.

32. Tan Cl, Kent GN, Randall AG, Edmondston SJ, Singer KP. Age-related changes in collagen, pyridinoline, and deoxypyridinoline in normal human thoracic intervertebral discs. J Gerontol A Biol Sci Med Sci. 2003;58:B387-93.

33. Allevi P, Cribiu R, Giannini E, Anastasia M. Practical syntheses of pyridinolines, important amino acidic biomarkers of collagen health. J Ora Chem. 2007;72:3478-83. 